\title{
Metrics Feedback Cycle: measuring and improving user engagement in gamified eLearning systems
}

\author{
Adam Atkins ${ }^{1}$, Vanissa Wanick ${ }^{2}$, Gary Wills ${ }^{3}$ \\ 1 First Author University of Southampton, adam@adamatkins.co.uk \\ 2 Corresponding Author University of Southampton, v.w.vieira@soton.ac.uk \\ ${ }^{3}$ University of Southampton, gbw@ecs.soton.ac.uk
}

\begin{abstract}
This paper presents the identification, design and implementation of a set of metrics of user engagement in a gamified eLearning application. The 'Metrics Feedback Cycle' (MFC) is introduced as a formal process prescribing the iterative evaluation and improvement of application-wide engagement, using data collected from metrics as input to improve related engagement features. This framework was showcased using a gamified elearning application as a case study. In this paper, we designed a prototype and tested it with thirty-six $(\mathrm{N}=36)$ students to validate the effectiveness of the MFC. The analysis and interpretation of metrics data shows that the gamification features had a positive effect on user engagement, and helped identify areas in which this could be improved. We conclude that the MFC has applications in gamified systems that seek to maximise engagement by iteratively evaluating implemented features against a set of evolving metrics.
\end{abstract}

Keywords: gamification, elearning, metrics;

\section{Introduction}

With advances in interactive technologies and the widespread adoption of games in education, the learning experience has been through constant transformation and eLearning has become a common supplementation to traditional schooling. In some cases, (e.g. distance learning), traditional schooling has been completely replaced, with the implementation of Massive Open Online Courses (MOOCs), with platforms like Coursera, and online tools like Moodle and Blackboard. It is in this context that gamification has emerged as a design strategy to increase user engagement. There is significant activity both in academic literature and in industry around gamification [1]. In fact, gamification techniques are spanning many domains, including health and fitness, social computing, and the workplace [2]. In education, gamification has been used to make learning more engaging and motivating for students. Research has reported positive results for the application of gamification in education, which encourages institutions to employ gamified strategies with their learning activities [3]. Although this shows an inspiring scenario for the adoption of gamification, there is still little research in the measurement of user engagement in gamified eLearning systems.

Gamification is the use of design components from games in non-gaming contexts to enhance user engagement [4] and motivate users to change their behaviour [5]. Hence, user engagement and motivation are the key outcomes of gameful system design. By definition, engagement is an active relationship between a consumer and a product or service, with engagement measured with metrics of recency, frequency, duration, virality and ratings [6]. Metrics from these categories can be used to form an 'E-score' (i.e. engagement score), which is a single number defining the level of user engagement. Engagement also has a strong relationship with user experience, since it reinforces affective meaning, interaction, feedback and user control, which culminates in a positive user experience [7]. However, gamification faces a big challenge since it is very difficult to maintain long-term relationships with consumers [8]; i.e. 'keeping engagement levels high' is hard, but is achievable through positive reinforcements and actions towards mastery.

Considering this, our paper focuses on the concept of user engagement, how gamification techniques can be used to increase user engagement, and how it is possible to measure the effectiveness of gamification techniques. More specifically, this paper presents the Metrics 
Feedback Cycle (MFC), which is a framework for using metric data to improve gamification features, with the aim of increasing user engagement within a gameful application. In order to explore the usage of the framework, we created a prototype for gamified eLearning, using gamelike elements to motivate students to learn mathematics. Details of the prototype development are discussed in this paper, together with the metrics implemented within the prototype, which measure the user engagement effect of corresponding gamification features.

It is important to mention, though, that together with gamification, there is the application of games in education (serious games), which are used for purposes other than entertainment. This paper focuses on gamification, as the emphasis is on the incorporation of game elements into an application, rather than creating a complete game. This paper concludes with recommendations for metrics and features in systems that employ gamification, as well as a prescription for further work on and usage of the Metrics Feedback Cycle (MFC) for systematically improving user engagement in other domains, over more practical longitudinal studies, and as part of software engineering processes, to enrich real-world applications.

\section{Theoretical background}

This section is an analysis of the main aspects of gamification in learning environments, including the relationship between gamification, engagement and motivation.

\subsection{Gamification in learning environments}

In learning environments, explicit rewards systems like points and levels are only one part of gamification. In other words, gamification in education goes beyond those elements, involving aspects like sense of engagement, immediate feedback, a feeling of accomplishment, and success of overcoming a challenge [9]. Unsurprisingly, education is one of the most common applications of gamification, with the aim to promote enjoyable experiences for learners through the use of levels, feedback and engaging challenges [2]. Two notable and successful examples are DuoLingo and Coursera, but some less intrusive examples of gamified learning are present in videogames like Minecraft [10] [11] [12].

As an implemented concept, gamification is advantageous in many business contexts, as it can be used to improve engagement with a product or brand [6], and facilitate long term product-user relationships by motivating users to interact with the gamified elements of the application/system (e.g. gain points in a reward system). Foursquare, StackOverflow and eBay are some examples of web companies using gamification techniques to improve user experience and engagement.

Understanding and quantifying the level of engagement in gamified applications is paramount when looking for ways to measure the effectiveness of gamification techniques. In fact, the main components of a gamified strategy are: the definition of outcome, user requirements and metrics [13]. Without a doubt, there is a need for metrics for measuring user engagement levels in gamified applications in education. This could lead to subsequent evaluation of gamification features with user engagement as criterion, followed by iteration on their design.

\subsection{Gamification and engagement}

The number of times users interact with an application, including the frequency and duration, gives an indication of user engagement [6]. This indication of engagement can be captured by a range of metrics. For example, if the application provides a way to interact with other people, such as giving tips, ratings and feedback, then engagement levels can be derived from the interactions that users have with this element. To truly capture long-term engagement levels, multiple metrics covering different aspects and time horizons are necessary, particularly because users may be very engaged during their first interaction and then abandon the application after some time. This approach is heavily used in measurements of website performance through tools like Google Analytics (GA), which can provide detailed data about the number of visits, including concepts like bounce rate, unique visitors per page, error page analysis, dropout rate and return visits [14]. This utilisation of $G A$ could be very helpful when looking for insights about interactions with web applications; however in the case of gamified applications it is expected that those metrics would be adapted. That is, user engagement is more than just clicks and interactions, it is about the user experience [7].

In gamified applications there is the concept of gamification analytics, which tends to seek out aspects that combine game elements and interactions with systems. An example of a game metric is 
the average time of completion of a level, the E-score (which measures recency, frequency, duration, virality and rating) and social game metrics, which is used to measure the daily active users (DAU), entry and exit factors, retention, interactions with other members and re-engagement (similar to return visits) [15]. What has not been mentioned in the work of $\mathrm{Xu}$ [15] are the other qualities of user engagement, such as feedback, interest and motivation, which can also help to measure the level of user engagement in gamified applications. Thus, considering this, there are motivational elements that should be considered when looking for metrics of effectiveness of gamified systems.

\subsection{Gamification and motivation}

Learning and motivation are two interrelated concepts, particularly in gaming environments. In games, players are required to learn the game's rules and continue playing in order to progress, feeling hooked and interested in particular game elements or mechanics through feedback loops [16]. For example, players can perceive the consequences of their actions within the gaming environment, augmented by their choices in the game. With this, learning becomes a motivating action, in which players can get a sense of accomplishment and learn by doing. In this scenario, games turn into effective tools to enhance learning motivation, which includes states like focus, engagement and enthusiasm over time [17]. Thus, when using gamification techniques in education, 'learning motivation' from a student's perspective must be considered, in order to guide gamification feature discovery.

Motivational affordances refer to implemented gamification features, such as a points system, leaderboards or badges. Psychological outcomes and behavioural outcomes are the dependent variables, and may relate to increased user engagement behaviour or increased motivation to succeed in the game/application. The focus of this paper is on motivational affordances, the resultant behavioural outcomes and their relation to user engagement. 'Behavioural outcomes' refers to the resultant change in user behaviour stemming from the psychological outcomes induced by the motivational affordance.

Some examples of motivational affordances described in [2] are a points system, leaderboards, badges/achievements, levels, having an overall story/theme, clear goals, feedback, rewards, progress and challenge. These motivational affordances focus on different areas of engagement, e.g. increasing engagement, providing instant feedback, or inducing a feeling of accomplishment.

The two types of motivation are intrinsic and extrinsic. Intrinsic motivation is the desire to perform an activity for the enjoyment of the activity itself, whereas extrinsic motivation is the desire to perform an activity solely for the enjoyment of the outcome of that activity [18] [8]. According to Hamari et al.[2], extrinsic motivation can deteriorate, depending on individual characteristics; thus, extrinsic motivation has to be combined with intrinsic motivation in order to change behaviour [19]. If using extrinsic rewards solely, then change of behaviour may only be temporary, before the original behaviour is reverted. For example, an individual may feel motivated to study hard, but after time the desire for the outcomes of studying hard may reduce, so the original behaviour of working less hard may return.

Squire [20] compares Pac-Man with traditional schooling and suggests that schools are structured around extrinsic motivators, such as good grades or fear of failure. In contrast: games are played for the intrinsic reward of playing them, and for the emotional state they induce [21]. Crossing over the two may have some benefit, as developing a learning system that evokes the same intrinsic reward and emotional response, may (at least partially) remove the need for extrinsic rewards, resulting in the learning application itself motivating students.

The origination of motivation for an individual can be derived from four main types of interaction. These include, 'Hard Fun', 'Easy Fun', 'Serious Fun' and 'People Fun' [22]. Table 1 shows commonly used gamification techniques (all used in this study), along with the effect on engagement and the type of interaction.

Considering this, there are many benefits of gamification, such as increased engagement, increasing the intrinsic reward produced by a learning system, reducing the need for extrinsic rewards, providing immediate feedback and nurturing a sense of accomplishment. 
Table 1. Commonly used gamification techniques, the resultant effect on engagement, and the type of motivation interaction involved

\begin{tabular}{|c|c|c|}
\hline $\begin{array}{l}\text { Gamification } \\
\text { technique }\end{array}$ & Effect on Engagement & Motivation interaction \\
\hline Leaderboard/Points & $\begin{array}{l}\text { Users return, to try and gain points } \\
\text { or progress in the leaderboard }\end{array}$ & $\begin{array}{l}\text { - 'People Fun' interaction } \\
\text { - Competitive nature of } \\
\text { leaderboard encourages users } \\
\text { to overtake their peers } \\
\text { - Points system without } \\
\text { leaderboard reduces potential } \\
\text { embarrassment while } \\
\text { maintaining a level of } \\
\text { competition, through indirect } \\
\text { social means }\end{array}$ \\
\hline Badges/Achievements & $\begin{array}{l}\text { Users return, to continue with a } \\
\text { challenge in order to receive a } \\
\text { badge or achieve a goal, increasing } \\
\text { engagement }\end{array}$ & $\begin{array}{l}\text { - } \quad \text { 'Easy Fun' interaction } \\
\text { Badge introduces a goal for } \\
\text { users to work towards }\end{array}$ \\
\hline Levelling system & $\begin{array}{l}\text { Users remain engaged for longer, } \\
\text { to try to progress to the next level }\end{array}$ & $\begin{array}{l}\text { - 'Hard Fun' interaction } \\
\text { - Defined levels act as goals for } \\
\text { - } \quad \text { user to work towards } \\
\text { Hard, as opposed to 'Easy } \\
\text { fun', as gaining levels can be } \\
\text { laborious }\end{array}$ \\
\hline Feedback & $\begin{array}{l}\text { When users receive positive } \\
\text { feedback their engagement } \\
\text { increases. Negative feedback has } \\
\text { potential to reduce engagement if } \\
\text { it occurs too often }\end{array}$ & $\begin{array}{l}\text { - 'Easy Fun' interaction } \\
\text { - Motivation to receive good } \\
\text { feedback over negative } \\
\text { - Can be linked to } \\
\text { badges/achievements }\end{array}$ \\
\hline
\end{tabular}

\subsection{Metrics of gamification effectiveness}

There is a breadth of research considering metrics for monitoring web applications [23], and features for increasing engagement, gamification-based and otherwise. However, currently there is no formal definition of a process describing the iterative evaluation and improvement of engagement features, using metric data as guidance. Therefore, there is an opportunity to link the two concepts of metrics and features in a useful and synergistic manner, using a feedback loop to positively reinforce features that promote desired behaviour.

Literature case studies by O'Donovan et al. [24] and Ibanez et al. [25] make use of gamification features for improving engagement, and touch on metrics to a lesser extent, but do not use the collected metric data for iterative improvement. O'Donovan et al. [24] looked at metrics from the perspective of the student through student questionnaires, feedback and other data analytics, such as attendance, grades and time spent, and Ibanez et al. [25] collected similar qualitative and quantitative data.

Similarly, Heilbrunn et al. [27] [28] utilised expert interviews to confirm that key performance indicators (KPIs), game elements and user groups are important areas in which metric data should be collected and monitored. These are the areas of focus in our study.

In terms of actual metrics, these are sometimes borrowed and adapted from web analytics, with metrics such as: bounce rate, churn, average revenue per user (ARPU) and daily active users (DAU) [15]. Commercial products like Google Analytics can be used to collect some of these metrics and provide broad insight, but are too generalised for specific feature-level monitoring. Heilbrunn et al. [28] imply that bespoke metrics are necessary to monitor custom application features. This paper focuses on bespoke metrics, often tailored per-feature, to track the engagement effect of implemented gamification features. These not only measure the engagement levels of the eLearning application, but also contribute to a novel way of improving gamified systems, by providing actionable analytics data. 


\section{Metrics Feedback Cycle (MFC)}

Following the definition of application-specific metrics and gamification features, the Metrics Feedback Cycle (MFC) is a reusable gamification architecture for iteratively improving engagement within an application. More precisely, the MFC is a systematic process involving implementation and evaluation of gamification features and metrics, with the target of the optimisation being the improvement of user engagement. The feedback cycle has some parallels with software development methodologies, such as the spiral model [28], where it can be easily integrated into the model's planning and development stages.

Figure 1 shows the formal MFC process:

1. Implement new metrics, adapt existing metrics, or remove metrics, to better describe engagement levels

2. Implement new features, revert to old feature set, or adapt existing features, to increase engagement levels or solve technical or user experience (UX) issues

3. Evaluate features using the defined metrics, potentially using both quantitative and qualitative metrics, which capture information from multiple areas/interactions/user sets within the application

4. Continue to step 1 or 2

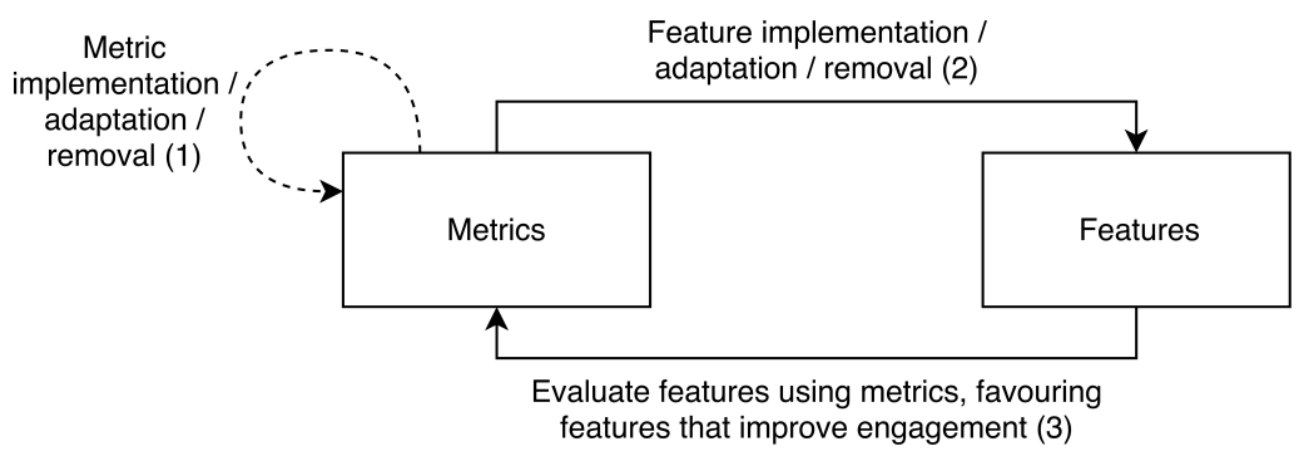

Figure 1 Formal graphical representation of the Metrics Feedback Cycle (MFC) for iteratively improving engagement within an application

As shown in Figure 1, the Metrics Feedback Cycle (MFC) is an iterative model, where improvements to the system are made through data analysis of a set of user engagement metrics. Since there is little research in this area, we apply the principles of the MFC in a case study, which is described in the next sections.

\section{Methodology and case study design}

This section describes the steps taken in the case study of identifying, implementing and evaluating the user engagement metrics and features to apply the Metrics Feedback Cycle (MFC) in the gamified system.

The study was conducted following four stages:

- Metrics and feature definition: identification of features to be deployed in the context of eLearning with the purpose of increasing engagement, and identification of corresponding metrics to measure engagement levels

- Metrics and feature implementation: the implementation of the metrics and features identified in the previous stage and in the prototype design

- User data storage implementation: a Firebase database [29] (NoSQL JSON tree) was used to store data from user interactions, to later derive metrics

- Evaluation: evaluation of features using the metrics identified and implemented in the previous stages with users. The Metrics Feedback Cycle (MFC) is followed through to one iteration

For the implementation of those four stages, we designed a prototype with gamified elements and implemented the Metrics Feedback Cycle (MFC). For the evaluation step of the MFC (step 3), we conducted a study with students, to evaluate engagement levels with the prototype. Thus, since 
our objective was to implement and evaluate the application of the MFC, the MFC process described in the previous section guided our methodology and study design. This is explained in detail in the next sections.

\section{Project background}

An existing eLearning web application was used, allowing rapid implementation of features and metrics with the intent to implement and evaluate the MFC. Figures 2, 3 and 4 show the paper selection homepage, the question screens, and the question with a wrong answer, alongside some gamification features (e.g. levels, badges). Users were invited to select a question paper (i.e. several questions, each broken down into multiple-choice sub-questions, guiding students through the solution) and complete it, and then return to the homepage to complete more papers. Users could leave the site at any time, without losing any data.

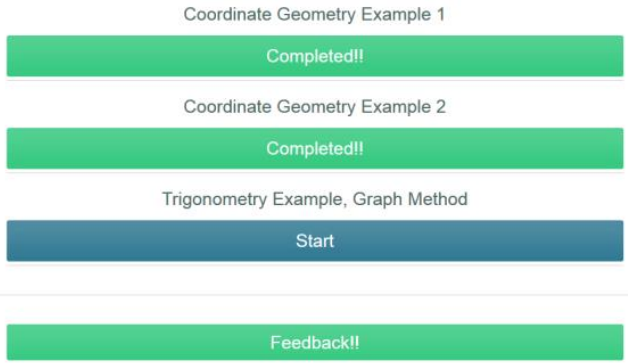

Figure 2 Homepage for accessing question papers

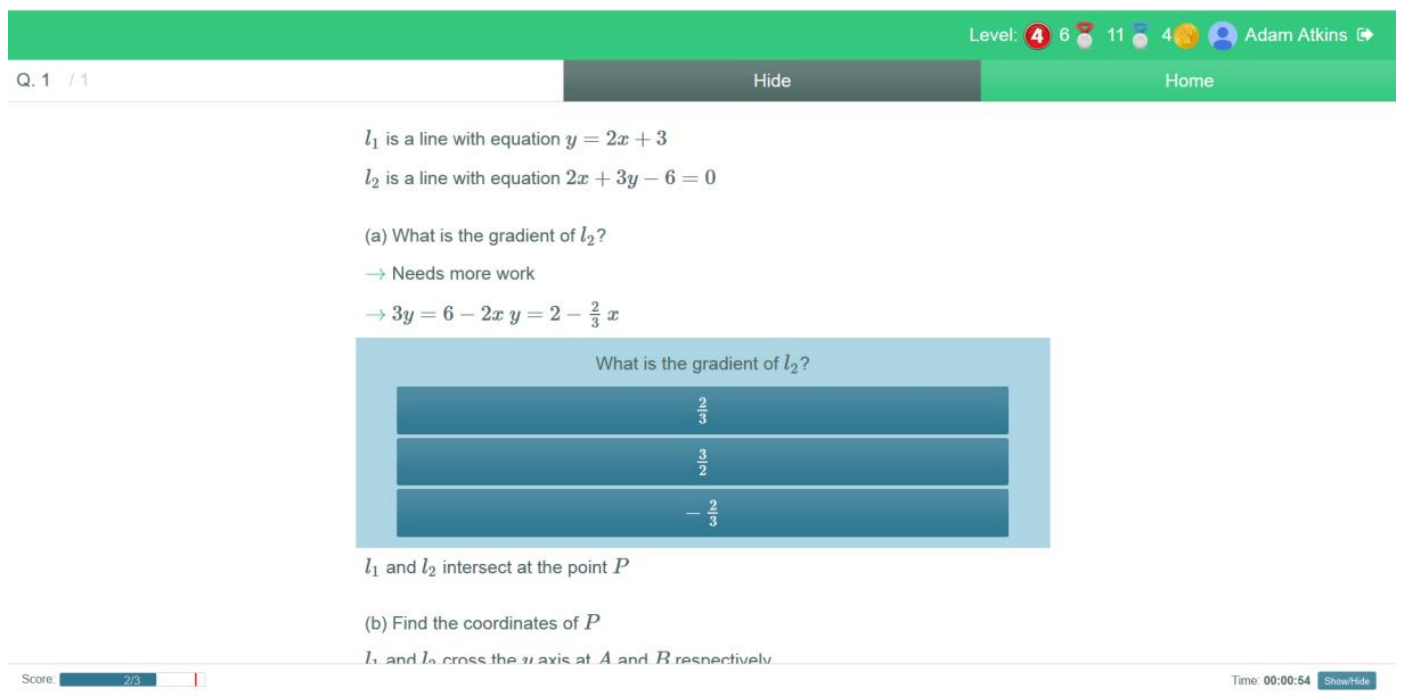

Figure 3 A question paper, also showing some of the implemented gamification features 


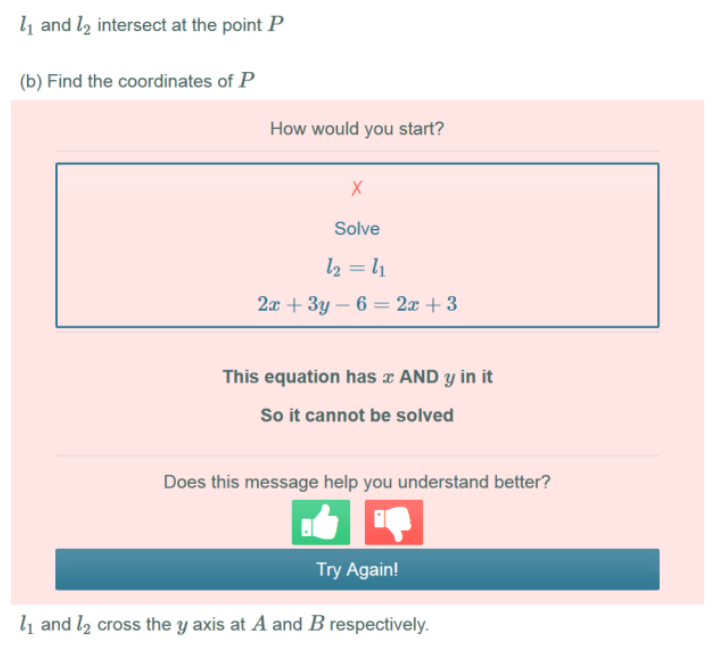

Figure 4 A question paper showing an incorrect answer

\subsection{Engagement metrics}

Since the main objective of the study was to measure the levels of user engagement in a gamified learning application, it was necessary to explore the elements that measure user engagement (see Table 2). The chosen user engagement metrics are a reflection of the purpose of the eLearning application, like motivating learners to engage with the content by giving them the opportunity to indicate their preference with a thumb signal, similar to forums like StackOverflow. These metrics are combined with the gamification features, which are described in the next section.

Table 2. Summary of User Engagement Metrics

\begin{tabular}{|c|c|c|}
\hline Metric & Definition & Explanation \\
\hline Dropout rate & $\begin{array}{l}\text { The number of questions } \\
\text { completed, divided by the } \\
\text { number of questions } \\
\text { attempted (completed or not), } \\
\text { for a user. This metric } \\
\text { captures a user's engagement } \\
\text { through a mixture of their } \\
\text { performance and their } \\
\text { persistence. }\end{array}$ & $\begin{array}{l}\text { A per session value, per user } \\
\text { Can be sliced and diced in multiple ways; e.g. get the } \\
\text { overall application engagement by summing dropout } \\
\text { rates over all users and sessions, or get the dropout rate } \\
\text { for a subset of users during various time periods, on } \\
\text { certain areas of the application. }\end{array}$ \\
\hline $\begin{array}{l}\text { Error message } \\
\text { display time }\end{array}$ & $\begin{array}{l}\text { The amount of time a user } \\
\text { spends reading the reason } \\
\text { why their answer was } \\
\text { incorrect. }\end{array}$ & $\begin{array}{l}\text { A useful metric for discovering which questions/topics } \\
\text { users are more or less interested in, due to the time they } \\
\text { spend reading the message popups. } \\
\text { This metric can then be used to adjust the way in which } \\
\text { questions on certain topics are presented, to improve } \\
\text { question quality and subsequently engagement. }\end{array}$ \\
\hline $\begin{array}{l}\text { Total time spent } \\
\text { vs. Total number } \\
\text { of questions } \\
\text { attempted }\end{array}$ & $\begin{array}{l}\text { For a user, the total session } \\
\text { time divided by the number of } \\
\text { questions attempted. }\end{array}$ & $\begin{array}{l}\text { Useful for comparing a user's engagement across } \\
\text { different sessions. A lower score means the user is } \\
\text { more active/engaged, and a higher score may indicate } \\
\text { distraction or lack of engagement. } \\
\text { Increase in user performance over time means this } \\
\text { metric is non-stationary, so it is harder to compare } \\
\text { across periods. } \\
\text { Comparisons between different users may be } \\
\text { inaccurate, as their abilities may differ. } \\
\text { Can be aggregated against topics to identify systematic } \\
\text { variation. }\end{array}$ \\
\hline Tips appreciation & $\begin{array}{l}\text { A thumbs up/down button is } \\
\text { available for the user to } \\
\text { indicate whether a tip } \\
\text { message is helpful or not. Tip } \\
\text { appreciation scores are stored } \\
\text { per question step, as tips }\end{array}$ & $\begin{array}{l}\text { Can be used to indicate whether particular types of tip } \\
\text { are more useful than others, and indicate question tips } \\
\text { that may be improved upon, to increase engagement. } \\
\text { Users may feel discouraged if tips aren't helpful. Tips } \\
\text { appreciation messages occur } 10 \% \text { of the time, so } \\
\text { they're not too intrusive. }\end{array}$ \\
\hline
\end{tabular}




\begin{tabular}{|c|c|c|}
\hline & $\begin{array}{l}\text { occur when a question step is } \\
\text { answered incorrectly. }\end{array}$ & \\
\hline $\begin{array}{l}\text { Proactive } \\
\text { feedback }\end{array}$ & $\begin{array}{l}\text { Qualitative feedback textbox } \\
\text { displayed to users after they } \\
\text { finish a paper, to allow them } \\
\text { to comment on how they } \\
\text { found the paper. This field is } \\
\text { optional, as forcing user } \\
\text { responses may introduce } \\
\text { noise. }\end{array}$ & $\begin{array}{l}\text { Useful to have direct feedback, alongside the indirectly } \\
\text { collected metrics, to corroborate any hypotheses } \\
\text { identified from the quantitative metrics. } \\
\text { A chance to indicate any flaws within the application } \\
\text { that weren't caught during testing or by other metrics. }\end{array}$ \\
\hline Bounce rate & $\begin{array}{l}\text { For a time slice, the number } \\
\text { of visits with no activity } \\
\text { divided by the total number of } \\
\text { visits. }\end{array}$ & $\begin{array}{l}\text { Measures the number of users who access the site and } \\
\text { immediately navigate away from it. } \\
\text { Bounce rates can be analysed when implementing new } \\
\text { application features, to gauge the level of interest. } \\
\text { Can be used as a tool to warn if the application is slow } \\
\text { to load, unresponsive or not rendering for a certain } \\
\text { device. } \\
\text { High bounce rate can prompt developers to perform } \\
\text { engagement analysis, to find improvements. }\end{array}$ \\
\hline Return visits & $\begin{array}{l}\text { For a time slice, the number } \\
\text { of users who visited the } \\
\text { application, who have } \\
\text { previously visited it in a } \\
\text { previous time slice. }\end{array}$ & $\begin{array}{l}\text { Provides an indication of the level of prolonged user } \\
\text { engagement: a large number of users returning in a } \\
\text { new time slice indicates high levels of prolonged } \\
\text { engagement with the application. } \\
\text { More advanced analysis can be conducted into the } \\
\text { number of slices users are active across before } \\
\text { becoming disengaged. }\end{array}$ \\
\hline
\end{tabular}

\subsection{Gamification features}

This section introduces the gameful features implemented in the system for motivating students to remain engaged in the eLearning activity. The background and rationale for each feature is discussed in each subsection.

\subsubsection{Levelling system}

A levelling system was deemed an advantageous feature addition given the educational application context because it encourages students to return, increase their level, gain more coins and overtake their friends. As described in Yee [30] and Smahel et al. [31], levelling systems work by giving the player gratification quickly at first, but then slowly increasing the distance between levels, making players work harder for rewards in a cyclic manner [32][33].

The levelling system comprises levels $0-9$, where a new player will start on level 0 and gradually work their way to level 9. The distance between each level increases, so higher levels are harder to attain. Experience (XP) is used to quantify the difference between levels, and their current progress, and can be seen on the rewards panel (see Figure 5). The better a student performs on a question, the more experience they gain. In addition, some questions have greater experience 'multipliers' as they are harder to complete.

The experience gained from a question paper is the user's score multiplied by a constant question difficulty multiplier. 8 is the maximum score, and the maximum question difficulty multiplier is 3 , resulting in a $24 \mathrm{XP}$ maximum. A score point is gained by answering a question correctly first time, and so is determined by the user's success on the question paper. Difficulty is a static multiplier, dependent on a question paper's perceived difficulty. It is decided by the teacher and ranges from 1 to 3 .

Currently, gaining levels does not grant the user access to new areas of the application, or access to harder questions offering more XP, although this would add another interesting aspect to the levelling user experience in a future version of the prototype.

Multiple mathematical experience functions were explored for determining the amount of XP between levels: exponential, polynomial, linear and Fibonacci. Linear was deemed too easy, as levels should be harder to attain as a student gains more levels, to indicate the greater value of higher levels. The parameters in standard polynomial/exponential functions were hard to tune, meaning the levelling system might be too aggressive, or too flat. 


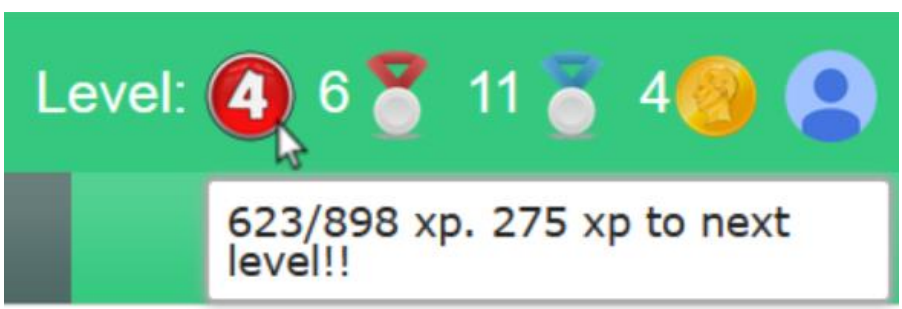

Figure 5 Rewards panel. (Left to right) Level with experience hover-over tooltip, number of trophies, daily login reward coins and logged in profile

\subsubsection{Fibonacci levelling system}

The Fibonacci function was deemed most appropriate for the levelling system, as it provides a challenging gradient at later levels without deterring lower level students. Although it is an exponential function, it is non-parametric, so only the start point of the series required tuning. Levels 1-9 are defined as $F(10)$ to $F(18)$ in the Fibonacci function, and level 0 is set to 0 experience. The start point was determined following ad-hoc calculations of how many questions students would need to complete to breach each level, based on the experience gained under each difficulty multiplier. It was necessary to shift the series in this way, to enforce steady level gains, so students are unable to jump multiple levels after answering one hard question. The Fibonacci sequence is summarised below:

$$
\begin{gathered}
F_{l}=F_{l-1}+F_{l-2} \\
\text { where } F_{0}=0, \quad F_{1}=1 \\
\text { e.g. } F_{0} \ldots F_{12}=0,1,1,2,3,5,8,13,21,34,55,89,144
\end{gathered}
$$

The starting point adjustment resulted in the following values from the Fibonacci experience function:

$$
\begin{aligned}
l_{0}=0, l_{0 \rightarrow 1}= & 55, l_{1 \rightarrow 2}=89, l_{2 \rightarrow 3}=144, l_{3 \rightarrow 4}=233, l_{4 \rightarrow 5}=377, l_{5 \rightarrow 6} \\
& =610, l_{6 \rightarrow 7}=987, l_{7 \rightarrow 8}=1597, l_{8 \rightarrow 9}=2584
\end{aligned}
$$

The above describes how much additional XP is required to reach the next level in each case, and 0 illustrates this.

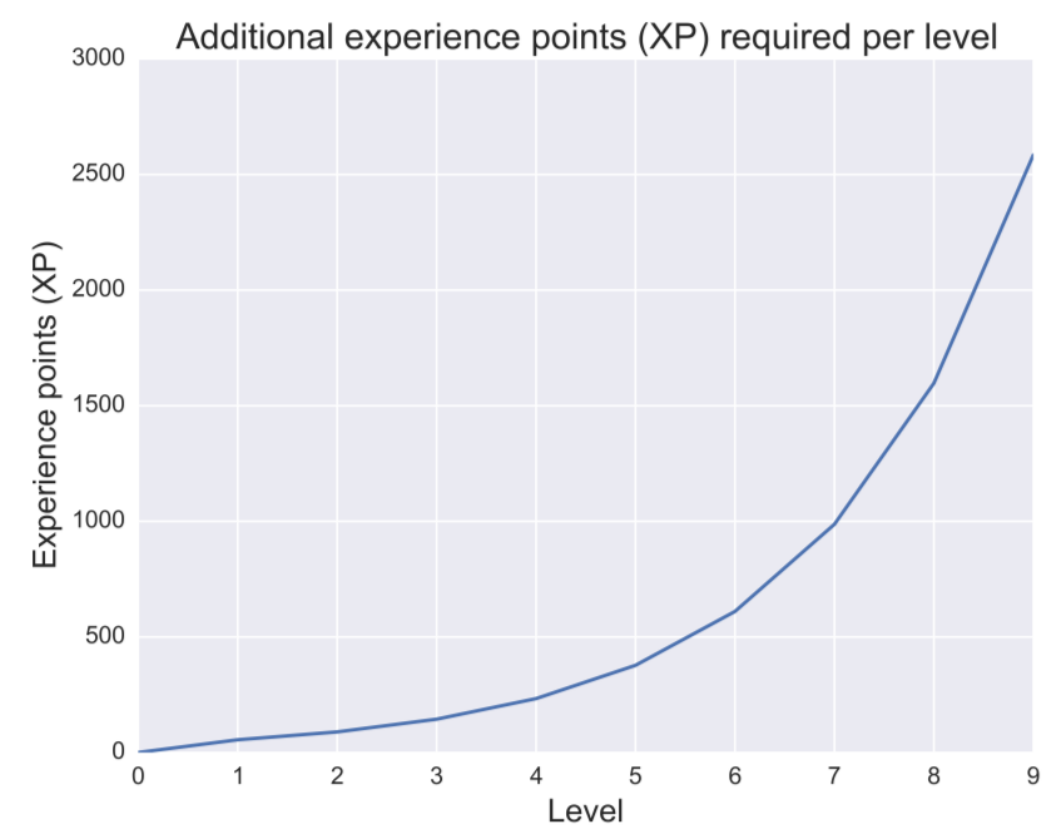

Figure 6 Fibonacci experience curve; shows the additional XP required to reach the next level from the previous. 


\subsubsection{Daily login rewards}

A gamified coin-based reward system was implemented, as another way to encourage students to return to the application (see 0). Similar to the levelling system and trophy rewards, it encourages progress within the application. In this case it encourages daily logins, which potentially leads students to attempt questions after logging in, and subsequently become more engaged in the application [32] [33]. The idea of implementing daily login rewards came about as many popular MMORPG games (such as Guild Wars, Warframe and Rift) use daily login rewards to increase engagement. Logging in can be seen as a threshold, that when crossed, elicits a spike in user engagement. This spike in engagement can be used to draw the user into other activities in the application, as the barrier to entry has been broken down.

A potentially necessary extension of daily login rewards is to only grant these when users carry out a number of actions, for instance completing a paper first, forcing engagement. This may help prevent the undesired behaviour of users logging off as soon as they receive the reward. Also, completing a paper may spark the student's interest and draw them in, through other gamified aspects.

\subsubsection{Trophy rewards}

A reward system distinct from 'levels' and 'login rewards' was implemented, with badges/trophies of different intrinsic value, depending on their attainment difficulty. The potential value of badgebased achievement systems is shown by Denny [34], where a large scale experiment was conducted whereby the period of user engagement in the application was extended without reduction in the quality of students' contributions. Qualitative data showed that students enjoyed having the ability to earn badges. Anderson et al. [35] refer to badges as offering a credentialing system, as well as an incentive system. Relating to the eLearning application, trophies are incentives to do well, and double up as credentials for comparing classmates to each other, both formally, through teachers, and informally, through social circles. The credential-incentive relationship is intertwined, as there is an incentive to improve one's own credentials in relation to their peers. The credentialing system is implicit in the school social environment and not explicitly defined in the application e.g. as a leaderboard. This is advantageous in an eLearning context, as it reduces potential direct embarrassment while maintaining a level of competition through indirect social means.

Other research in [36] provided the motivation to implement trophies, as they were used in a very similar eLearning context, and proved a relatively successful gamification technique in their case.

Using trophies alongside a levelling system and daily login rewards provides a richer user experience than using any one of these reward systems purely in isolation. Intuitively, users are more interested in a game that has multiple measures of success and multiple facets to explore.

Two types of medals are defined: blue and red. 'Blue medals' are won merely by completing question papers successfully. 'Red medals' are more valuable, and are awarded each time a user completes a paper with full marks. The medals are shown in the rewards panel, alongside level and login rewards $(0)$.

\subsubsection{Health bar and ghost of past self}

Introducing a health bar was a natural addition to the gamification feature set, as it's a gaming concept most people are familiar with. It has potential to encourage students into a playful mind-set, rather than strictly a work mind-set, as they may have come across health bars in gaming contexts before. The health bar is an indicator of how well a student is performing on a particular question paper. Its fill is the number of correct answers divided by the total answers given. Health bars can be used to determine a state and are dynamic, depending on the interaction the user has with the game or system [37]. In other words, a health bar is usually a visualisation of the gamification concept of 'progress'. In games, a health bar can also represent the effectiveness of a player's strategy and can measure achievements, such as winning a match [38].

Rather than displaying a percentage that students might associate with exams, which may be intimidating, the health bar adds playfulness to the user experience (see 0). Also, a percentage score may wrongly suggest to the student the marks they would expect to receive in a test, which might not be the case, as the application is not under exam conditions. 


\section{Score: 1}

Figure 7 Health bar with ghost of past self. Appears in bottom panel.

To build upon this, when students come back to a question paper, their past attempt is indicated on the health bar as a red line. This encourages students to compete with themselves, by trying to surpass their previous final health score. Self-competition was favoured over implementing a feature to evoke competition between users, which may discourage weaker students from participating, due to potential embarrassment.

\subsubsection{Question timer}

As students approach exams, a useful feature is to be able to time question answering, so exam readiness can be tracked. As well as the obvious utility benefits of the tool, it is also an instrument for gamification, as it encourages self-competition, albeit less directly than the health bar.

Question timing and test score data can be aggregated and used as engagement measures, effectively using student performance as a proxy for engagement. Improvement in question completion time and test scores indicates a more engaged learner.

The timer does not affect a student's final score, and it can optionally be hidden, so that students are not encouraged to rush through papers to complete them quickly (see 0 ). Functionally, the ethos of the application is around students learning from their mistakes by reading the tips, and less around speed, until they're confident.

\section{Time: 00:36:44 Show/Hide}

Figure 8 Question timer with show/hide. Appears in bottom panel.

\subsubsection{Distraction-reduction alerts}

As the application is web-based, there is a possibility of users changing tabs or applications, due to distractions. To maintain a high level of engagement, it is important to regain the user's attention when their focus is not on the application. To achieve this, the web page title blinks between two messages when focus is on another tab or application. After 15 seconds of the application being out of focus, the title intermittently changes (every half second) between two predefined strings 'Hey!' and 'Come back!'. An alert sound also plays after the 15-second period of inactivity, but only once, to avoid potential annoyance.

\subsubsection{Random encouragements}

Web-based tutoring loses some of the nuances of traditional tutoring, such as encouragement and motivation provided by a human tutor, which stimulates the student, and in turn reinforces their engagement and concentration (see 0). To try and replicate this to some extent, random encouragement messages are displayed to the user when they answer a question, $5 \%$ of the time.

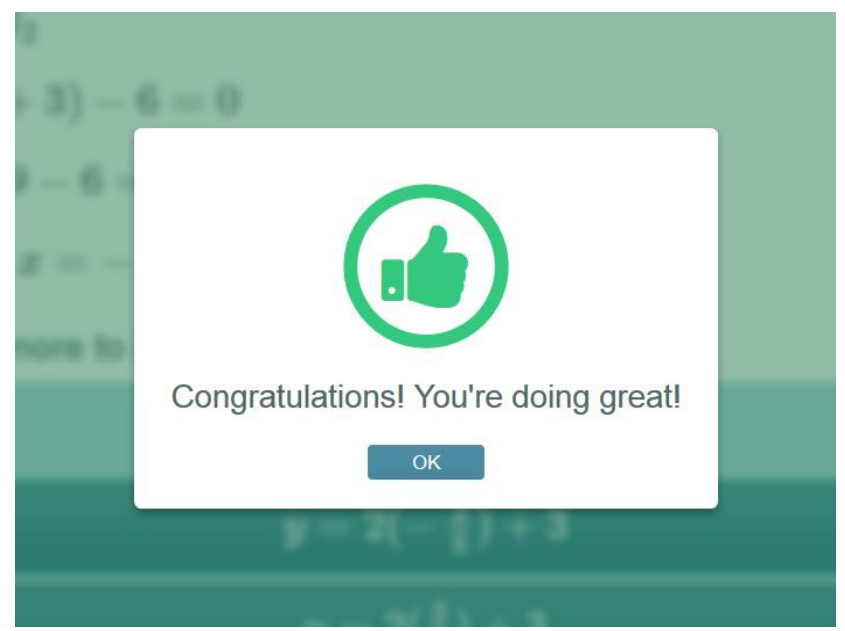

Figure 9 A random encouragement message 
This method of increasing engagement is distinct from levels, and trophy rewards, in that it benefits both low and high achieving students. The other gamification features may alienate lower achieving students, whereas random encouragements (along with 'Blue trophy rewards') remedy this, as they are related to effort rather than performance.

The existing encouragement mechanism is rudimentary; however more targeted encouragement messages in later versions could potentially increase engagement further. For example, 'You're doing really well at topic X. Keep up the good work!', which may be triggered because the student has improved significantly over a number of papers in a given topic. Furthermore, encouragements like these can be displayed when a student positively completes a task, rather than displaying them randomly; this could be more effective for overall student motivation. Currently, the dialog is modal, but in later versions a notification style popup will be used, to ensure it doesn't interrupt the student's train of thought.

\section{Data analysis and interpretation}

Thirty-six $(n=36)$ participants were invited to take part in the study. All participants had, or were working towards, advanced level high school mathematics qualifications and were between 16-18 years old. Participants were both male and female, although demographic data was not recorded, as considering differences between sexes is not the purpose of this study. Participants were invited to interact with the online prototype and solve three question papers in one, approximately one hour, session. The sessions took part under guided supervision, in a quiet classroom. Data from the participants' interaction were gathered during this process and assigned to an anonymous string ID, so no personal information was recorded.

Error Message Display Time

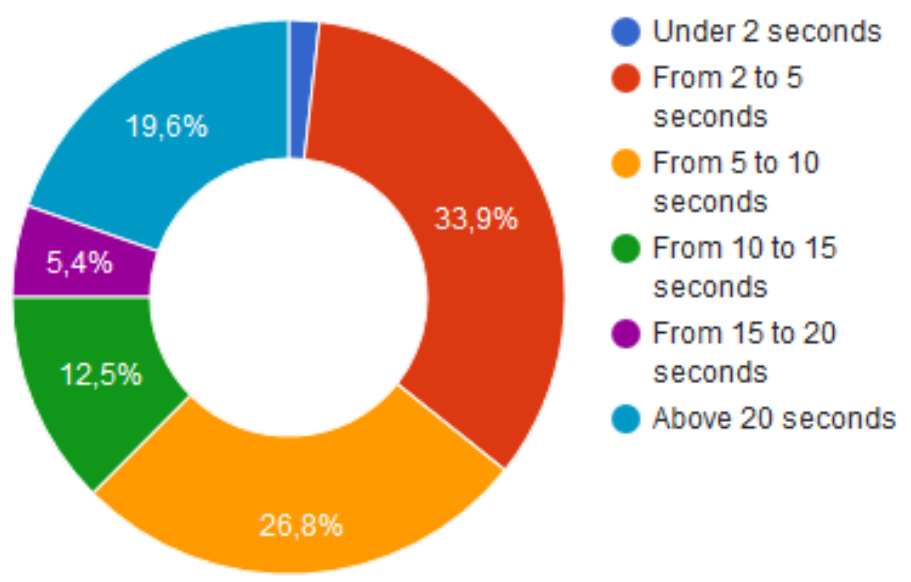

Figure 10 Error message display time 
Tips Appreciation by Question Paper

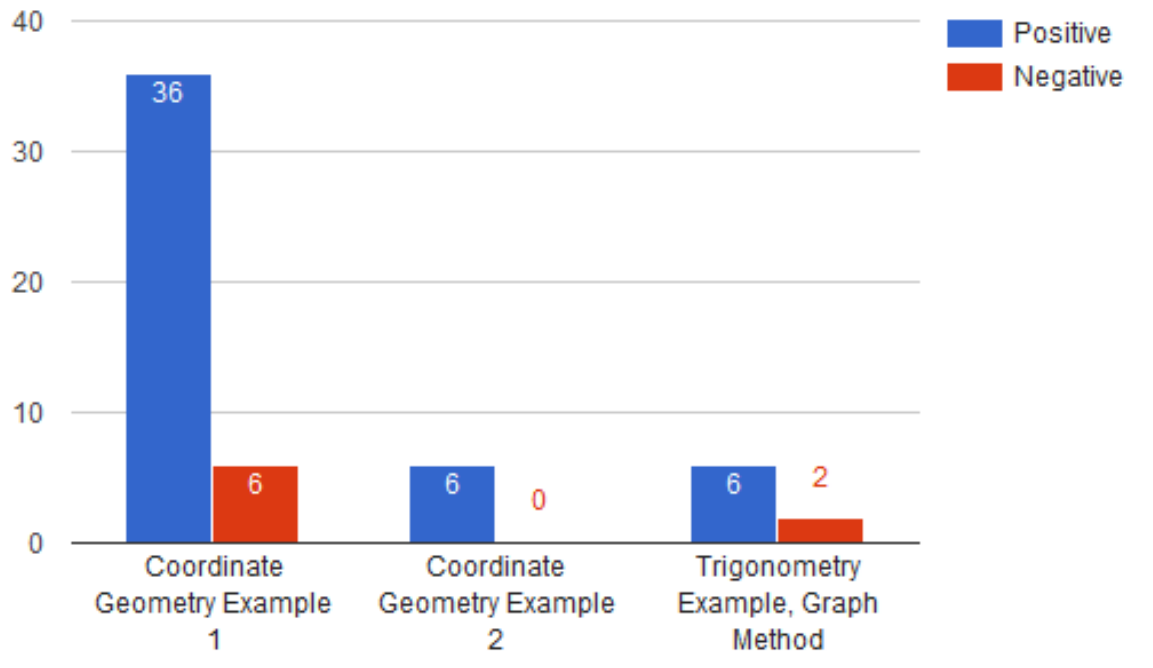

Figure 11 Tips appreciation by question paper

Proactive Feedback by Question Paper

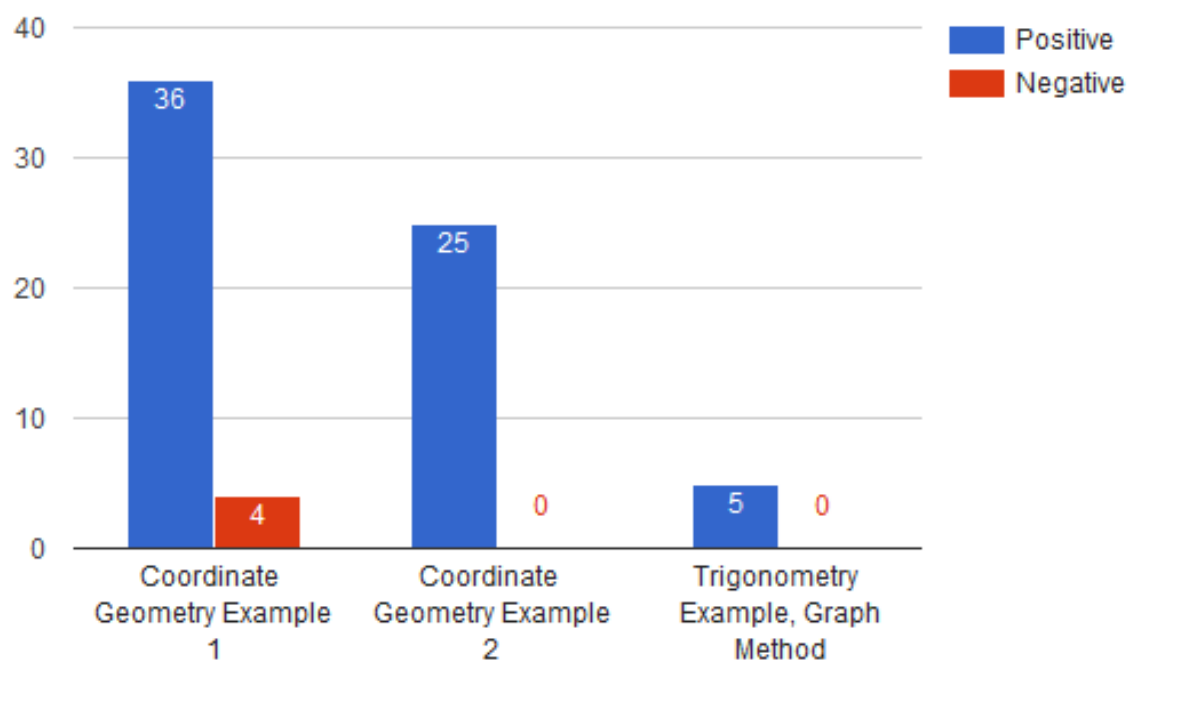

Figure 12 Proactive feedback by question paper

0 shows that $64.3 \%$ of error messages were displayed for over 5 seconds on screen, indicating that most users engaged with the error messages, and found them useful. Only $1.8 \%$ were displayed for less than 2 seconds, which is deemed short for a user to read and absorb, and is an encouragingly small percentage of users who did not engage with the error message fully. It may be useful to conduct further analysis into the engagement level of the group of $33.9 \%$ users who viewed the error message for less than 5 seconds, as this group appears uninterested in the error message and likely made little use of the help. Following prolonged application usage, the engagement level of this group can be analysed over time to determine whether it systematically varies due to question type, topic or other factors, which may help in adjusting features to engage these students. However, a portion of this group is likely composed of noise, due to users miss-clicking the incorrect answer.

The high percentage of users who read the error messages suggests that the feature is a useful one for increasing engagement and provides students with valuable information. This is indicated by the median display time of 6.9 seconds, alongside the fact that almost $20 \%$ of users read the 
messages for over 20 seconds. Both the error message feature, and associated timing metric, proved useful when evaluated in accordance with the Metrics Feedback Cycle (MFC), so should be kept in the next version.

The metrics collected around tips appreciation show $85.7 \%$ of students voting positively, with the remainder not finding these useful (see 0). This data is shown at paper level, but when more users are involved, patterns may emerge around the kind of tips students appreciate more than others, which can in turn guide the feature enhancement process. This can also be a simple way of identifying erroneous tip messages that need fixing, which is likely to occur when more papers are introduced. A potential drawback of the tips appreciation mechanism is that the modal nature of the dialog may force students to quickly press either 'thumbs up' or 'thumbs down' to make the dialog disappear. This would skew the results upwards, as people may be subconsciously more inclined to pick the 'thumbs up' as a default to dismiss the modal window, as the icon has positive connotations. A solution for more accurate data is to make this feedback optional, rather than enforced.

Relating back to the Metrics Feedback Cycle (MFC), the tips appreciation metric can be used to filter out and remove tip messages that are not appreciated, and implement more of the type of tips that students are fond of. In this case we are using 'appreciation' as a proxy for engagement, with the assumption that students are more engaged when they appreciate a tip message on a question.

In terms of the proactive feedback feature, and the associated metrics derived from the qualitative data, shown in 0 , we can see that the feedback was mostly positive $(94.3 \%$ of feedback positive). However, negative feedback has the potential to provide just as much value as positive feedback, as this can be used to adjust features accordingly. For example, some feedback stated the question was 'very clear and helpful' which is encouraging, but less useful. Others pointed out that a generated image was not showing, which was input into a backlog for the next iteration of feature implementation, following the MFC. Although this is a usability issue, it is associated with engagement, as poor usability can lead to frustration and disengaged users.

\section{Discussion}

The set of metrics were helpful for finding issues and potential improvements, but more importantly they provided information on the usage and appreciation of some of the application's key features. This use of metric data in order to improve gamification features has not been employed before and this is the first study that utilises this approach. The Metrics Feedback Cycle (MFC) showed a starting point for the usage of data as a means for personalisation and user experience optimisation in gamified systems. Data collected during the MFC study revealed scope for personalisation of gamification features based on user interaction, which is an important strategy that requires an understanding of user preferences, and can even be tied to personality traits [39]. In this case, the MFC also demonstrated that gamified learning environments should be adaptive and dynamic, including not only interactions with the application but also the levels of user engagement. That is, the MFC measures engagement in a holistic way that was not measured in detail before in previous research [40].

The present case study was prone to some limitations. Firstly, although our research showed the application of the Metrics Feedback Cycle (MFC) in a small-scale study, on a subset of the implemented features and metrics, a longitudinal study could be very useful to understand other metrics that were implemented but not utilised in this study, and may help further demonstrate the benefits of the MFC. For instance, tracking daily login rewards over a longer time period would help determine the effectiveness of this re-engagement feature for drawing users back to the application. Secondly, other ways to record user interaction could have been used. For instance, it would be useful to record user interaction with the rewards panel, which contains their level, trophies and daily login rewards and subsequently a vast part of the gamification interaction. More engaged students may generally check experience points and the rewards panel more frequently, so these could be another set of metrics for determining user-wide engagement in the application, especially when adapting/adding rewards panel features. Coupled with other engagement metrics in the 'Metrics Feedback Cycle', it can be used to determine the effect new features have had on engagement, potentially reducing these to a single score, like the 'E-score' (i.e. single number that measures engagement for a feature, or set of features) [6].

In the same way as the rewards panel 'E-score', the 'distraction reducing alert' metrics can be distilled into a single numerical value for ease of use. A future metric implementation in a 
longitudinal study could measure how long it takes for the user to return after the alert sounds, together with how often users would be distracted from the application.

Alongside improvements to the metrics, and ways in which to aggregate them, the overall gameful experience could be improved by introducing more gaming elements, such as missions, mini-games and the introduction of an overall theme (e.g. medieval, pirates or sci-fi). These game mechanics target user intrinsic motivation, whereas others, like virtual goods or gifting mechanisms, are more extrinsically oriented [12][40][41].

\section{Conclusions}

This paper shows the identification, implementation and evaluation of user engagement metrics and features in a gamified learning environment. The main contribution of this paper is the Metrics Feedback Cycle (MFC), which is a unique and formal definition of a process for iteratively improving engagement within an application, by using metrics data to systematically improve engagement features. The case study allowed the framework to be used and evaluated in a real-world eLearning context, with gamification as the focal instrument for improving engagement. Usage of the MFC encourages user engagement to be measured in a holistic way, considering user interactions with the entire system as well as with the gamified elements. This can be extremely useful when exploring the linkage between personalisation and gamification.

An analysis of gamification literature identified the linkage between gamification and engagement, and how it can be used as a tool for increasing engagement, and more broadly a tool for changing behaviour. Understanding how users might interact with different kinds of features was fundamental to the feature discovery process, and techniques like categorising features into 'People fun', 'Easy fun', 'Serious fun' and 'Hard fun' groups guided this process [22]. 'People/Easy/Hard fun' features were the focus, while avoiding the 'Serious fun' category in this case study.

The implemented gamification features were discussed, along with design decisions made; such as the levelling function intensity, as tuning this has a distinct engagement effect, so was carefully selected. The features implemented are well known and widely used in academia and industry; however, the novelty lies in selection of context appropriate features that provide a suitable overall gamification experience, and identifying corresponding metrics that marry up with these features appropriately.

The results of the study show that the users were engaged in the application, so the implemented features are proven to be useful, where data is available: students responded positively towards the error messages, tips, and the proactive feedback. Further student interaction across a longer time span is necessary to fully make use of the Metrics Feedback Cycle (MFC), as this would allow more data points to be collected, allowing for richer analysis.

A drawback of the study is that it is not longitudinal, with returning students, so it was not possible to show multiple iterations of the Metrics Feedback Cycle (MFC) and subsequent modification of the features and metrics at each stage. This also left some of the metrics redundant, as they are appropriate to multiple visits. For example, 'Dropout rate' and 'Total time spent vs questions attempted' pertain to multiple sessions as their purpose is for making comparisons across sessions, and identifying any systematic variation of engagement levels - e.g. certain topics or questions may be linked to low engagement sessions. Further work in the area would involve making use of the Metrics Feedback Cycle (MFC) in a longitudinal study, to evaluate its efficacy more fully. Exploring more formal linkage to software development models, like the spiral model, is also an area of future focus, as gamification needs to be fully integrated and proven within development processes in order to encourage its wider use.

The features and metrics identified are of use in eLearning related contexts, but more broadly, the Metrics Feedback Cycle (MFC) has applications in any context that seeks to maximise engagement by iteratively evaluating and adapting implemented features against a set of evolving metrics, within a wider software engineering process. 


\section{References}

[1] Adkins, S., "The 2016-2021 Global Game-based Learning Market," in Serious Play Conference, 2016.

[2] Hamari, H., Koivisto, J., Sarsa, J., "Does Gamification Work? - A Literature Review of Empirical Studies on Gamification," in System Sciences (HICSS), 2014 47th Hawaii International Conference on, 2014, pp. 3025-3034. https://doi.org/10.1109/HICSS.2014.377

[3] Dicheva, D., Dichev, C., Agre, G. and Angelova, G., "Gamification in Education: A Systematic Mapping Study," J. Educ. Technol. Soc., vol. 18, pp. 75-88, 2015.

[4] Deterding, S., Sicart, M., Nacke, L., O'Hara, K. and Dixon D., "Gamification. using gamedesign elements in non-gaming contexts," in the 2011 annual conference extended abstracts, 2011, pp. 2425-2428. https://doi.org/10.1145/1979742.1979575

[5] Deterding, S., "Situated motivational affordances of game elements : A conceptual model," in CHI2011, 2011, pp. 3-6.

[6] G. Zichermann and C. Cunningham, "Gamification by Design: Implementing Game Mechanics in Web and Mobile Apps," in Gamification by Design: Implementing Game Mechanics in Web and Mobile Apps, O'Reilly Media, Inc, 2011, p. 208.

[7] O'Brien, H. L and Toms, E. G., "What is user engagement? A Conceptual Framework for defining user engagement with technology," J. Am. Soc. Inf. Sci. Technol., vol. 59, no. 6, pp. 938-955, 2008. https://doi.org/10.1002/asi.20801

[8] AlMarshedi, A., Wanick, V., Ranchhod, A., and others, "SGI: A framework for increasing the sustainability of gamification impact,” Int. J. Infonomics, vol. 8, no. 1/2, pp. 1044-1052, 2015. https://doi.org/10.20533/iji.1742.4712.2015.0123

[9] Kapp, K. M., "The Gamification of Learning and Instruction: Game-based Methods and Strategies for Training and Education," in The Gamification of Learning and Instruction: Gamebased Methods and Strategies for Training and Education, San Francisco: John Wiley \& Sons, 2012, pp. 1-22.

[10]I. R. M. Association, Gamification: Concepts, Methodologies, Tools, and Applications, 1st ed. Hershey, PA, USA: IGI Global, 2015.

[11] Gee, P. J., "Good video games and good learning," in Phi Kappa Phi Forum, 2005, vol. 85, no. 2, p. 33.

[12] Squire, D., “'All I Know I Learned from Zelda': Immersive Gaming and Learning and Why The Legend of Zelda Is a Perfect Learning Game," in Learning to Play: Exploring the Future of Education with Video Games, M. Khine Swe, Ed. Peter Lang, 2011, pp. 167--176.

[13] Deterding, S. "The Lens of Intrinsic Skill Atoms: A Method for Gameful Design," HumanComputer Interact., vol. 30, no. 3-4, pp. 294-335, 2015. https://doi.org/10.1080/07370024.2014.993471

[14]Clifton, B., Advanced Web Metrics with Google Analytics. 2010.

[15]Xu, Y., "Literature Review on Web Application Gamification and Analytics," 2011.

[16] Gee, J. P., "Learning by design: Games as learning machines," Interact. Educ. Multimed., vol. 8, pp. 15-23, 2004.

[17] Garris, R., Ahlers, R. and Driskell, J. E. ,"Games, Motivation, and Learning: A Research and Practice Model," Simul. Gaming, vol. 33, no. 4, pp. 441-467, Dec. 2002. https://doi.org/10.1177/1046878102238607

[18] Ryan, R. M. and Deci, E. L. "Intrinsic and Extrinsic Motivations: Classic Definitions and New Directions," Contemp. Educ. Psychol., vol. 25, no. 1, pp. 54-67, 2000. https://doi.org/10.1006/ceps.1999.1020

[19] AAlmarshedi, A., Wanick, V., Wills, G., and Ranchhod, A., "Gamification and Behaviour," in More than just games, Stieglitz et al. (eds.), Ed. Switzerland: Springer International Publishing, 2016, pp. 19-29.

[20] Squire, K., "Video games in education.," Games Simul., vol. 2, no. 1, pp. 49-62, 2003.

[21] Herz, J. C., Joystick Nation: How Videogames Ate Our Quarters, Won Our Hearts, and Rewired Our Minds, 1st ed. Boston, MA: Little, Brown \& Co. Inc., 1997.

[22] Lazzaro, N., "Why We Play Games : Four Keys to More Emotion Without Story," 2004.

[23] A. Phippen, L. Sheppard, and S. Furnell, "A practical evaluation of Web analytics," Internet Res., vol. 14, no. 4, pp. 284--293, 2004. https://doi.org/10.1108/10662240410555306

[24] O'Donovan, S., Gain, J., Marais, P., Donovan, S. O. and Marais, P., "A Case Study in the Gamification of a University-level Games Development Course," in ACM International Conference Proceeding Series, 2013, pp. 242-251. https://doi.org/10.1145/2513456.2513469 
[25] Ibanez, M.-B., Di-Serio, A., and Delgado-Kloos, C., "Gamification for Engaging Computer Science Students in Learning Activities: A Case Study," IEEE Trans. Learn. Technol., vol. 7, no. 3, pp. 291-301, 2014. https://doi.org/10.1109/TLT.2014.2329293

[26] Heilbrunn, B., Herzig, P. and Schill, A., "Towards gamification analytics-requirements for monitoring and adapting gamification designs," 44th Annu. Meet. Soc. Comput. Sci. INFORMATICS 2014, vol. P-232, pp. 333-344, 2014.

[27] Heilbrunn, B., Herzig, P. and Schill, A., "Tools for gamification analytics: A survey," in Proceedings - 2014 IEEE/ACM 7th International Conference on Utility and Cloud Computing, UCC 2014, 2014, pp. 603-608. https://doi.org/10.1109/UCC.2014.93

[28]Boehm, B. W., “A spiral model of software development and enhancement," Computer (Long. Beach. Calif)., vol. 21, no. 5, pp. 61-72, 1988. https://doi.org/10.1109/2.59

[29] Google, "Firebase." [Online]. Available: https://firebase.google.com/.

[30] Yee, N., "Ariadne - Understanding MMORPG Addiction," 2002. .

[31] Smahel, D., Blinka, L. and Ledabyl, O., "Playing MMORPGs: connections between addiction and identifying with a character.," Cyber Psychol. Behav., vol. 11, no. 6, pp. 715--718, 2008. https://doi.org/10.1089/cpb.2007.0210

[32] Wen, D. M.-H., Chang, D. J.-W., Lin, Y.-T., Liang, C.-W., and Yang, S.-Y. "Gamification design for increasing customer purchase intention in a mobile marketing campaign app," in International Conference on HCI in Business, 2014, pp. 440-448. https://doi.org/10.1007/9783-319-07293-7_43

[33] Meder, M., Plumbaum, T., and Hopfgartner, F., "Perceived and actual role of gamification principles," in Proceedings of the 2013 IEEE/ACM 6th International Conference on Utility and Cloud Computing, 2013, pp. 488-493. https://doi.org/10.1109/UCC.2013.95

[34] Denny, P. “The Effect of Virtual Achievements on Student Engagement," in Proceedings of the SIGCHI Conference on Human Factors in Computing Systems, 2013, pp. 763-772.

[35] Anderson, A., Huttenlocher, D., Kleinberg, J., and Leskovec, J.“ Steering User Behavior with Badges," in Proceedings of the 22Nd International Conference on World Wide Web, 2013, pp. 95-106.

[36] Domínguez, A., Saenz-de-Navarrete, J., de-Marcos, L., Fernández-Sanz, L., Pagés, C. , and Martínez-Herráiz, J.-J., "Gamifying learning experiences: Practical implications and outcomes," Comput. Educ., vol. 63, pp. 380-392, 2013. https://doi.org/10.1016/j.compedu.2012.12.020

[37] Pinchbeck, D., “An affordance based model for gameplay,” Break. New Gr. Innov. Games, Play. Pract. Theory Proc. 2009 Digit. Games Res. Assoc. Conf., 2009.

[38] Salen, K. and Zimmerman, E., Rules of Play: Game Design Fundamentals, vol. 37, no. 5. Massachusetts Institute of Technology, 2004.

[39] Tondello, G. F., Wehbe, R. R., Diamond, L., Busch, M., Marczewski, A. and Nacke, L. E. "The Gamification User Types Hexad Scale,” Proc. 2016 Annu. Symp. Comput. Interact. Play - CHI Play '16, pp. 229-243, 2016. https://doi.org/10.1145/2967934.2968082

[40] Monterrat, B., Lavoué, É. and George, S. "Motivation for learning: Adaptive gamification for web-based learning environments," in CSEDU 2014 - Proceedings of the 6th International Conference on Computer Supported Education, 2014, vol. 1, pp. 117-125.

[41] Malone, T. W., "What makes things fun to learn? heuristics for designing instructional computer games," in Proceedings of the 3rd ACM SIGSMALL symposium and the first SIGPC symposium on Small systems - SIGSMALL '80, 1980, pp. 162-169. https://doi.org/10.1145/800088.802839

[42] Muntean, C. C. I., "Raising engagement in e-learning through gamification," 6th Int. Conf. Virtual Learn. ICVL 2011, no. 1, pp. 323-329, 2011. 